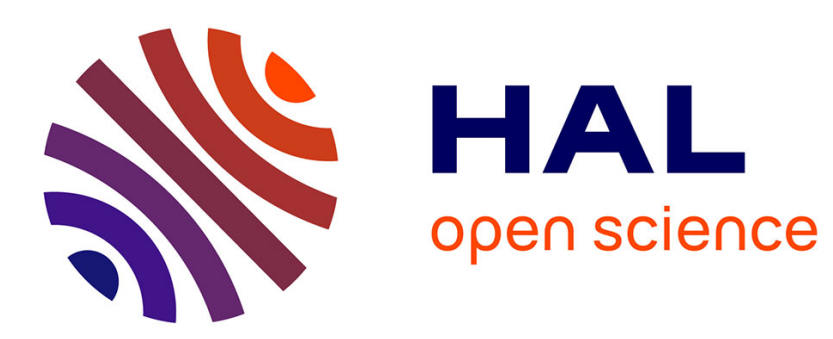

\title{
Phase-Coherent Heterodyne Detection in the Terahertz Regime With a Photomixer
}

\author{
Florin Lucian Constantin
}

\section{To cite this version:}

Florin Lucian Constantin. Phase-Coherent Heterodyne Detection in the Terahertz Regime With a Photomixer. IEEE Journal of Quantum Electronics, 2011, 47 (11), pp.1458-1462. 10.1109/JQE.2011.2163152 . hal-03335686

\section{HAL Id: hal-03335686 \\ https://hal.science/hal-03335686}

Submitted on 23 Sep 2021

HAL is a multi-disciplinary open access archive for the deposit and dissemination of scientific research documents, whether they are published or not. The documents may come from teaching and research institutions in France or abroad, or from public or private research centers.
L'archive ouverte pluridisciplinaire HAL, est destinée au dépôt et à la diffusion de documents scientifiques de niveau recherche, publiés ou non, émanant des établissements d'enseignement et de recherche français ou étrangers, des laboratoires publics ou privés. 


\title{
Phase-coherent heterodyne detection in the terahertz regime with a photomixer
}

\author{
Florin Lucian Constantin
}

Laboratoire PhLAM, CNRS UMR 8523, 59655 Villeneuve d'Ascq, France

\begin{abstract}
A LTG-GaAs photomixer with spiral antenna has a quadratic response to the optical fields and an intrinsic nonlinear IV characteristic that allow detection of $\mathrm{THz}$ radiation. A transmission line coupled to the antenna provides direct-current access to the photomixer in the MW domain. The non-ohmic response of the photomixer is probed by rectification. The optical beat between two cw lasers illuminating the photomixer serves as a local oscillator with an adjustable difference-frequency in the $\mathrm{THz}$ domain. The beat between a $\mathrm{THz}$ field modulated at a radiofrequency rate with a radiofrequency-modulated optical beat is exploited in a phase-sensitive detection scheme.
\end{abstract}

Keywords : Submillimeter-wave measurements, photomixer, rectification, heterodyne detection.

\section{Introduction}

Heterodyne detection is a well-established technique in the terahertz domain brought in the 1970s by high speed Schottky diodes. The idea [1] was to mix a $\mathrm{THz}$ source with a reference source (local oscillator, LO) in a nonlinear device to yield a signal at an intermediate frequency. Increased interest for frequency mixing in the $\mathrm{THz}$ regime is actually due for the applications in remote sensing technology, high-speed telecommunication and high-purity microwave signal generation. Recent progress with quantum cascade lasers allowed transposing a terahertz signal onto an optical carrier by a nonlinear optical mixing technique [2] and high-speed direct-current modulation [3].

The optical heterodyne conversion [4] in a photomixer irradiated by two $\mathrm{CW}$ near-infrared lasers is a well-established technique for the generation of monochromatic $\mathrm{THz}$ radiation. The standard photomixer design is a metalsemiconductor-metal device that drives a planar antenna. Upon the photomixer is biased, the optical beat between the lasers detuned in the THz domain lead an ultra-fast response in the electrical circuit that is radiated by the antenna. Optimisation of the semiconductor parameters or the antenna design [5] ensures operation over a wide frequency bandwidth. High-speed modulation from telecom lasers was transferred from the optical to the THz domain [6]. Conversely, the photomixer has been used as receiver with a homodyne detection scheme [7]. The THz-wave generated by optical heterodyne conversion of two 
lasers on a photomixer is frequency-converted to dc on a second photomixer using the optical beat of the same lasers. This detection technique is essentially monofrequency and forbid addressing the exceptional frequency bandwidth of the photomixer.

This paper reports a heterodyne detection scheme with a photomixer for an independent $\mathrm{THz}$ source of radiation. The optical beat between two lasers with a difference-frequency in the $\mathrm{THz}$ domain is the LO. The electric field induced in the antenna by the incoming $\mathrm{THz}$ beam is applied onto the conductance of the photomixer modulated by the optical beat. Quadratic response to the optical fields and the curvature of the current-voltage dependence of the photomixer allows nonlinear mixing and lead to signals at sum and differencefrequency. Tuning the frequency of the $\mathrm{THz}$ source or the optical beat allows accessing the low frequency signal with microwave instruments. This signal is sensitive to the amplitude and the phase of the THz field. Moreover, the geometry of the antenna allows frequency-selective detection of a spatial mode and polarisation of the incident $\mathrm{THz}$ field. In addition, the photomixer design allows applying an external microwave voltage over the antenna electrodes. This allows investigating through rectification the behaviour of the photomixer nonlinear conductance, a key issue for the heterodyne detection.

\section{Experimental setup}

The experimental setup (Fig. 1.A, Fig. 2.A) involves two extended-cavity diode lasers (DL 1, DL 2) in the Littrow configuration emitting around 820 $\mathrm{nm}$ and operated in a free-running mode. The photomixer uses a $2 \mu \mathrm{m}$ thick GaAs layer grown at low temperature (LTG-GaAs) on a GaAs semi-insulating substrate. A pattern of five $\mathrm{Ti} / \mathrm{Au}$ interdigitated electrodes (1.8 $\mu \mathrm{m}$ gap, 0.2 $\mu m$ width, over $8 \times 8 \mu m^{2}$ photoconductive area) are forming Schottky contacts with the LTG-GaAs layer. The electrodes are placed at the driving point of a self-complementary log-spiral antenna with an outer radius of $1.5 \mathrm{~mm}$. Laser beams with equal power and similar polarisation are spatially overlapped on a beamsplitter and focused on the electrodes with an aspheric lens.

An active frequency multiplier (Militech AMC-10-R0000) is used as the $\mathrm{THz}$ source. Output in the $75-110 \mathrm{GHz}$ frequency range with $\mathrm{mW}$ power level is acquired by multiplying 6 times a microwave $(M W)$ synthesizer. A separate synthesizer $\left(R F_{T H z}\right)$ can modulate the microwave. THz radiation emerging from a standard waveguide is focused on the antenna with a $10 \mathrm{~mm}$ diameter high-resistivity hyperhemispherical silicon lens mounted on the rear of the photomixer chip.

The outer extremities of the antenna are connected with wire bonds to a semirigid microwave transmission line ( $50 \Omega$ characteristic impedance) terminated with a bias- $T$ in order to address microwave $(A C$ port) and low-frequency ( $D C$ port) response of the photomixer. A synthesizer $\left(R F_{P m i x}\right)$ is coupled on the $A C$ port to probe the photomixer response. The bias voltage is applied through a RL choke on the $D C$ port. The $D C$ port is terminated with a capacitively-coupled resistive charge. This corresponds to the input impedance of a spectrum analyser $(S A)$ used to measure low-frequency changes of the photomixer current (Fig. 1.A). Alternatively, the measurement of heterodyne 
beatnotes is performed with $S A$ on the $A C$ port. In addition, a synthesizer $\left(R F_{L O}\right)$ can modulate a laser diode (Fig. 2.A). All synthesisers and the spectrum analyser have $50 \Omega$ internal impedance and are phase-referenced on a quartz oscillator. The measurements performed are corrected by the cable and connector losses, but not for the connexion losses of the photomixer to the semirigid transmission line, impedance mismatches and optical coupling losses.

\section{AC response of the photomixer circuit probed by rectification}

In the standard optical heterodyne theory, a difference-frequency signal is generated inside the photomixer by the quadratic response of the photoconductor to the optical fields and propagates to the measurement instrument. Conversely, in the heterodyne detection, a signal from an external source is coupled to the photomixer and modulates its conductance. This is allowed by intrinsic nonlinear mechanisms $[8,9,10]$ that govern the conduction in LTG GaAs photomixers. Typical DC current-voltage plot for the photomixer measured at low tension is shown in (Fig. 1.B). The sub-linear behaviour around zero-bias with significant second-order derivative will be investigated for detection purpose.

The microwave rectification [11] approach is used to probe the nonlinear response of the photomixer irradiated with one laser and biased at constant voltage. When a microwave signal is applied on the $A C$ port, rectification leads to a DC change of the current. A qualitative approach is the small-signal regime where the rectified current can be written as:

$$
I_{r}(\omega)=\frac{1}{2} I^{\prime \prime}(\omega) V_{\mu}^{2}(\omega)
$$

where $I^{\prime \prime}$ is the second derivative of the IV curve and $V_{\mu}$ is the amplitude of the microwave voltage across the device. The intrinsic transport mechanism is responsible for the frequency dependence of $I^{\prime \prime}(\omega)=I_{d c} /\left[1+(\omega \tau)^{2}\right]$ by taking in account its DC-value $I_{d c}$ and the carrier lifetime $\tau$. The actual value $(\tau=1.2$ ps) yields negligible frequency dependence in the microwave range. Frequency dependence of $V_{\mu}$ is governed by the circuit-dependent elements: intrinsic resistance of the device, reactance and loss of the antenna, parasitic coupling of the photomixer with the transmission line. For a given generator power $P_{\text {out }}$, the amplitude of the microwave voltage across the device is:

$$
V_{\mu}^{2}(\omega)=8 R_{0} P_{\text {out }} \beta(\omega)
$$

with $\beta(\omega)$ a circuit-dependent factor and $R_{0}=50 \Omega$ the generator internal resistance. $\beta(\omega) \rightarrow 1$ at the near-DC limit because of the high dynamic resistance of the photomixer $\left(R_{d}>>R_{0}\right)$ and the reflection of the microwave. The measurement of the rectified current is performed by amplitude modulation of the microwave. The current induced at the modulation frequency goes through the low-pass filtering DC path. The amplitude of the rectified voltage, measured at the modulation frequency with SA is divided by its $R_{0}=50 \Omega$ input impedance to yield the rectified current. 
Fig. 1.C displays the dependence of the rectified current against the microwave power at $2 \mathrm{GHz}$ for a photomixer biased at $-55 \mathrm{mV}$ under $20 \mathrm{~mW}$ optical power. A linear dependence is observed when the rectified current is small comparing with $\sim 2 \mu \mathrm{A}$ device DC current. The small-signal approximation is valid for a microwave power up to $\sim 1 \mathrm{~mW}$. The inset displays the level of the rectified current (reference $0.29 \mu \mathrm{A}$ ) from $1 \mathrm{~mW}$ microwave power. The signal to noise ratio (SNR) is $32.5 \mathrm{~dB}$ within a resolution bandwidth $\mathrm{RBW}=1$ $\mathrm{kHz}$.

The laser power dependence of the rectified current is linear (Fig. 1.D). The carriers contributing to the rectification process are therefore photo-generated and $I^{\prime \prime}(\omega)$ is proportional to the laser power. The rectified current can be estimated using Eq. (1) with the value of $I^{\prime \prime}(\omega)$ calculated by numerically differentiating data of the current-voltage plot and Eq. (2) at the low-frequency limit $(\beta(\omega)=1)$. The inset displays the voltage dependence of the measured and the calculated rectified current at $2 \mathrm{GHz}$. The values differ by one order of magnitude. This inconsistency can be attributed to parasitics in the microwave coupling to the photomixer. The discrepancy in the voltage dependence of the rectified current can be attributed to laser beam shape superposition with the microwave field over the photoconductive area and may point out that only a part of the DC current contributes effectively to rectification.

In addition, rectification in the photomixer circuit was measured by optically coupling a $\mathrm{THz}$ wave to the antenna. The approach followed is similar to the small-signal approximation developed for the MW regime. The $\mathrm{THz}$ generator is the equivalent-model Thévenin source $V_{T H z}(\omega)$ with the internal resistance $Z_{A}$ and the rectified current is:

$$
I_{r}=\frac{1}{2} I^{\prime \prime}(\omega) V_{T H z}^{2}(\omega) .
$$

with

$$
V_{T H z}^{2}(\omega)=2 Z_{A} P_{T H z}(\omega) \lambda(\omega) .
$$

where $P_{T H z}$ the $\mathrm{THz}$ power available from the multiplier and $\lambda$ is an antennadependent factor corresponding to the part of $P_{T H z}$ that is intercepted by the antenna.

Fig. 1.E displays the frequency dependence of the rectified current for the photomixer biased at $-55 \mathrm{mV}$ under $20 \mathrm{~mW}$ optical power. Experimental data is superposed with the theoretical curve for $I^{\prime \prime}(\omega)$ frequency dependence. The antenna time constant yields negligible frequency dependence in the range investigated here. The fluctuations observed are attributed to the frequency response of the coupling of $\mathrm{THz}$ power to the antenna. The inset displays the level of the rectified current (reference $0.1 \mu \mathrm{A}$ ) at $106.1 \mathrm{GHz}$. The signal to noise ratio is $20 \mathrm{~dB}$ within a RBW $=1 \mathrm{kHz}$. No calibration of the $\mathrm{THz}$ power was performed and the maximum value specified by the manufacturer $\left(P_{T H z}=2.5 \mathrm{~mW}\right)$ is attributed to the maximum rectified current $\left(I_{r}=0.4 \mu \mathrm{A}\right)$. The responsivity for the direct detection with the photomixer is estimated at $1.6 .10^{-4} \mathrm{~A} / \mathrm{W}$. The rectified current is negligible to the device DC current $\left(I_{d c}=2 \mu \mathrm{A}\right)$. Using the small-signal approximation Eq. (3), $I_{r}$ is calculated with the value of $I^{\prime \prime}(\omega)$ given by numerically differentiating data of the DC current-voltage plot at the operating voltage. For a photomixer biased at $-55 \mathrm{mV}$ under $20 \mathrm{~mW}$ optical 
power, $I^{\prime \prime}(\omega)=50 \mu \mathrm{A} / \mathrm{V}^{2}$ and rectification at $98.4 \mathrm{GHz}$ with a power estimated at $0.7 \mathrm{~mW}$ leads to $I_{r}=0.1 \mu \mathrm{A}$. The value of the $\mathrm{THz}$ power coupling factor to the antenna is estimated with Eq. (4) at 0.04. $\lambda$ exhibits voltage dependence due to similar reasons as those proposed for the rectification in the microwave regime.

\section{Heterodyne detection with a photomixer}

A qualitative approach can be derived for the heterodyne detection assuming that the photomixer acts as a rectifier in the small-signal regime. The optical beat induces a current at the difference-frequency $\omega$ and the subsequent voltage:

$$
I_{\text {diff }}(\omega)=2 R_{\text {opt }}(\omega) \sqrt{m P_{1} P_{2}} ; V_{\text {diff }}(\omega)=R_{d}(\omega) I_{\text {diff }}(\omega) .
$$

with $R_{\text {opt }}$ optical responsivity and $R_{d}$ dynamic resistance of the photomixer for a given voltage bias and under the combined laser powers $P_{1}, P_{2}$. The overlapping of the laser beams over the photoconductive area is accounted through the factor $m$. The optical difference-frequency voltage mixes with the antenna-induced $\mathrm{THz}$ voltage yielding the heterodyne current:

$$
I_{h}(\delta \omega)=\frac{1}{2} I^{\prime \prime}(\omega) V_{d i f f}(\omega) V_{T H z}(\omega+\delta \omega) .
$$

at a frequency $\delta \omega$ in the microwave range $\delta \omega<<\omega$.

Fig. 2.B shows the heterodyne beatnote level recorded on the $A C$ path between the two-laser $\mathrm{LO}$ and the $\mathrm{THz}$ source emitting $\sim 0.9 \mathrm{~mW}$ at $100.1 \mathrm{GHz}$. The photomixer is biased at $40 \mathrm{mV}$ under $2 \times 20 \mathrm{~mW}$ combined optical power leading to a heterodyne current of $2.8 \mu \mathrm{A}$. The signal-to-noise ratio (SNR) is 25 $\mathrm{dB}$ within $\mathrm{RBW}=1 \mathrm{MHz}$. The responsivity for the heterodyne detection with the photomixer is estimated at $3 \cdot 10^{-3} \mathrm{~A} / \mathrm{W}$. The half-linewidth at $-3 \mathrm{~dB}$ of the signal is $\sim 1 \mathrm{MHz}$. This is consistent with the linewidth of the beatnote from the two-laser LO detuned in the RF range and corresponds to the spectral purity of a laser.

At low frequency, the heterodyne voltage dependence governed by circuitdependent factors is neglected. The beatnote from the two-laser LO at a frequency difference of $\sim 0.8 \mathrm{GHz}$ under $5 \mathrm{~V}$ bias and a combined optical power of $2 \times 20 \mathrm{~mW}$ corresponds to a heterodyne current of $56.4 \mu \mathrm{A}$. From the total 110 $\mu \mathrm{A}$ DC current, the overlapping factor is estimated at $\mathrm{m}=0.26$. An estimation of the heterodyne current is performed from Eq. (4) and Eq. (5). $R_{o p t}$ and $R_{d}$ display a roll-off at high frequency due to the conduction mechanism, while the antenna time constant has negligible contribution. Within the experimental conditions from Fig. 2.B, $R_{\text {opt }}=5.10^{-5} \mathrm{~A} / \mathrm{W}, R_{d}=36 k \Omega$ and $I^{\prime \prime}(\omega)=9$ $\mu A / V^{2}$, that yields $V_{\text {diff }}=36 \mathrm{mV}$ and $V_{T H z}=72 \mathrm{mV}$ using the value $\lambda=0.04$. Eq. (6) leads to a heterodyne current $I_{h}=12 \mathrm{nA}$ which is two orders of magnitude less than the measured value. This discrepancy is attributed to a violation of the small-signal approximation, as the $1 \mu \mathrm{A}$ difference-frequency current is comparable with the $2 \mu \mathrm{A}$ DC current.

The photomixer is used further in a multi-heterodyne mixing scheme (Fig. 2.C). Frequency modulation (FM) of the MW synthesizer leads to a frequency comb 
that is subsequently up-converted in the THz domain. The heterodyne mixing with the two-laser $\mathrm{LO}$ down-converts the $\mathrm{THz}$ comb as shown in Fig. 2.C-2. The value of the FM index at $\mathrm{THz}$ frequency is estimated at 2.4 from the FM sensitivity of the MW synthesizer. A two-laser LO frequency comb is generated by direct-current modulation of one laser. Its frequency-domain spectra display phase-coherent and equally spaced sidebands with a dominant contribution from the frequency modulation. A phase-sensitive mixing scheme is demonstrated with the photomixer to cancel the frequency comb structure in the beatnote. The LO comb should be generated using the same modulation frequency as for the THz comb (Fig. 2.C-3). The amplitude and the phase of the modulation signal from $R F_{L O}$ are adjusted until sidebands are annulled as shown in Fig. 2.C-4.

\section{Conclusion}

This work exploits the nonlinear current-voltage response of the photomixer for detection of $\mathrm{THz}$ radiation. Rectification in the small-signal regime allows qualitative interpretation of the experimental results. The model should be improved for quantitative consistency with the results by taking into account the spatial dependence of MW and $\mathrm{THz}$ signals within the optical absorption depth of the LTG-GaAs layer and a high-signal approach for the heterodyne detection. Signals at $\sim 0.1 \mathrm{THz}$ with $\mathrm{mW}$-level power were detected through rectification with typical SNR of $20 \mathrm{~dB}$ within $1 \mathrm{kHz}$ RBW. Heterodyne detection with $\mathrm{SNR}=25 \mathrm{~dB}$ with $\mathrm{RBW}=1 \mathrm{MHz}$ was performed at $100.1 \mathrm{GHz}$. Enhancement of the sensitivity of the heterodyne detection can be provided by reducing the linewidth of the lasers. In addition, the phase-coherent multi-heterodyne mixing scheme, demonstrated between a THz and a two-laser LO frequency comb, could be extended over the broad frequency span available within the photomixer bandwidth.

\section{Acknowledgment}

The fabrication of photomixers and the measurements of the LTG-GaAs electron lifetimes were performed at Centrale de Technologie de l'IEMN. Financial support for this work from Ministère de l'Enseignement Supérieur et de la Recherche and CNRS is acknowledged. 


\section{References}

[1] H. R. Fetterman, B. J. Clifton, P. E. Tannenwald, and C. D. Parker, "Submillimeter detection and mixing using Schottky diodes," Appl. Phys. Lett., vol. 24, pp. 70-72, 1973.

[2] S. S. Dhillon, C. Sirtori, J. Alton, S. Barbieri, A. D. Rossi, H. Beere, and D. Ritchie, "Terahertz transfer onto a telecom optical carrier," Nat. Photonics, vol. 1, pp. 411-415, 2007.

[3] S. Barbieri, W. Maineult, S. S. Dhillon, C. Sirtori, J. Alton, N. Breuil, H. E. Beere, and D. A. Ritchie, "13 GHz direct modulation of terahertz quantum cascade lasers," Appl. Phys. Lett., vol. 91, pp. 143510 1-3, 2007.

[4] I. S. Gregory, C. Baker, W. R. Tribe, I. V. Bradley, M. J. Evans, E. H. Linfield, A. G. Davies, and M. Missous, "Optimisation of photomixers and antennas for continous-wave terahertz emission," IEEE J. Quantum Electr., vol. 41, pp. 717-728, 2005.

[5] E. R. Brown, F. W. Smith, and K. A. McIntosh, "Coherent millimeterwave generation by heterodyne conversion in low-temperature-grown gaas photoconductors," J. Appl. Phys., vol. 73, pp. 1480-1484, 1993.

[6] M. Martin, J. Mangeney, P. Crozat, Y. Chassagneux, R. Colombelli, N. Zerounian, L. Vivien, and K. Blary, "Gigahertz modulation of tunable terahertz radiation from photomixers driven at telecom wavelengths," Appl. Phys. Lett., vol. 93, pp. 131112 1-3, 2008.

[7] S. Verghese, K. A. McIntosh, S. Calawa, W. F. DiNatale, E. K. Duerr, and K. A. Molvar, "Generation and detection of coherent terahertz waves using two photomixers," J. Appl. Phys., vol. 73, pp. 3824-3826, 1998.

[8] E. R. Brown, K. A. McIntosh, F. W. Smith, K. B. Nichols, M. J. Manfra, C. L. Dennis, and J. P. Mattia, "Milliwat output levels and superquadratic bias dependence in a low-temperature-grown gaas photomixer," Appl. Phys. Lett., vol. 64, pp. 3311-3313, 1994.

[9] J. P. Ibbetson and U. K. Mishra, "Space-charge-limited currents in nonstoichiometric gaas," Appl. Phys. Lett., vol. 68, pp. 3781-3783, 1996.

[10] N. Zamdmer, Q. Hu, K. A. McIntosh, and S. Verghese, "Increase in response time of low-temperature-grown GaAs photoconductive switches at high voltage bias," Appl. Phys. Lett., vol. 75, pp. 2313-2315, 1999.

[11] H. C. Liu, J. Li, M. Buchanan, and Z. R. Wasilewski, "High-frequency quantum-well infrared photodetectors measured by microwave-rectification technique," IEEE J. Quantum Electr., vol. 32, pp. 1024-1028, 1996. 

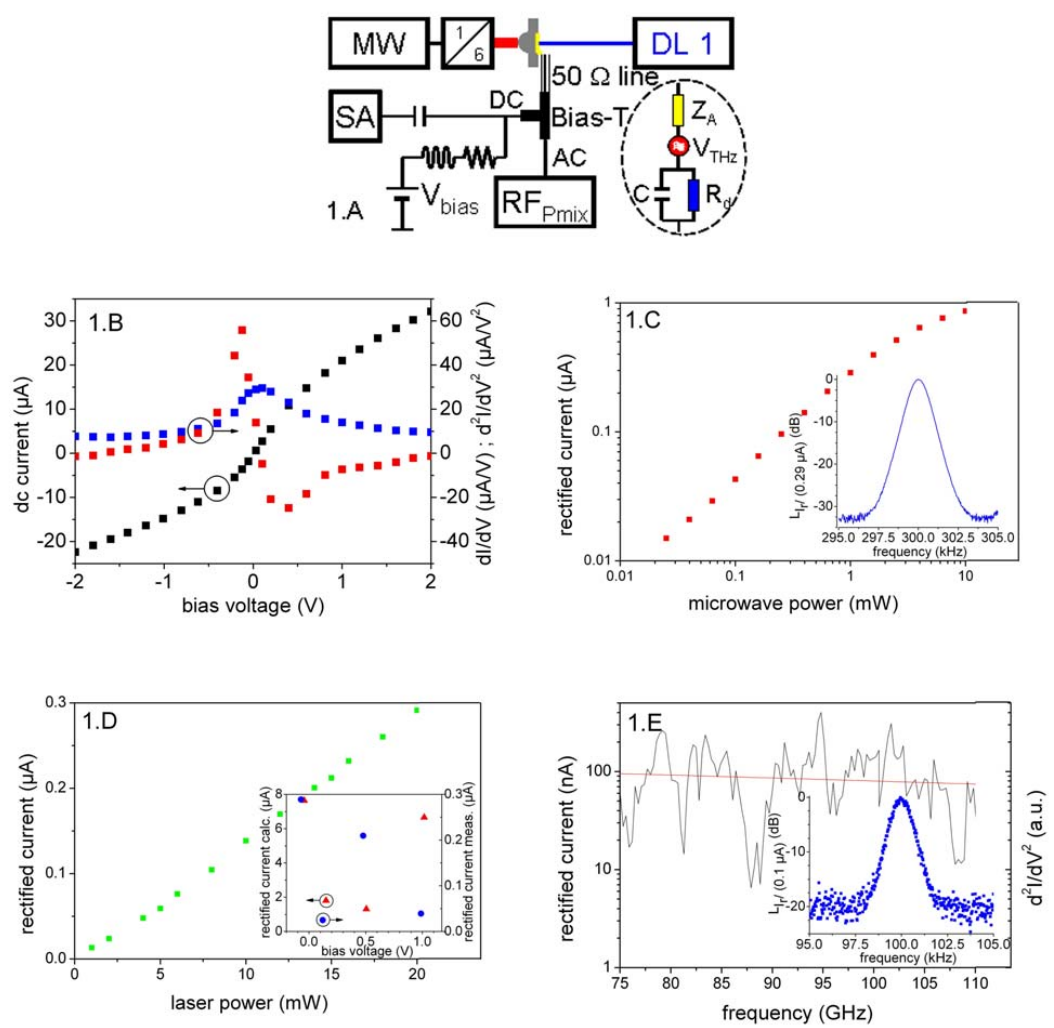

Figure 1: A. Characterisation of the electrical response. Inset: The photomixer equivalent-model circuit: an optically-driven nonlinear resistance $R_{d}$, shunted by a capacitance $\mathrm{C}$ (estimated at $0.5 \mathrm{fF}$ ) is in series with the antenna impedance $Z_{A}$ and its Thévenin-equivalent generator. $Z_{A}=72 \Omega$ is real at $\mathrm{THz}$ frequencies, and its frequency dependence is complex in the MW range with the obvious limit $Z_{A}(\omega \rightarrow 0) \rightarrow 0 \Omega$. LTG-GaAs electron lifetime $\tau=1.2$ ps and antenna time constant $2 \pi Z_{A} C=0.2$ ps.

B. DC-response under $20 \mathrm{~mW}$ laser power: current-voltage plot and first and second-order derivative calculated by numerical differentiation.

C. Microwave power dependence of the rectified current at $2 \mathrm{GHz}$. $R F_{\text {Pmix }}$ is $100 \%$ amplitude modulated at $300 \mathrm{kHz}$. Inset: Level of the rectified current (reference $0.29 \mu \mathrm{A}$ ), $1 \mathrm{~mW}$ microwave power. $\mathrm{RBW}=1 \mathrm{kHz}$, sweep time 275 ms, 50 video averages.

D. Laser power dependence of the rectified current: microwave power $1 \mathrm{~mW}$ at $2 \mathrm{GHz}$, optical power $20 \mathrm{~mW}$, voltage bias $-55 \mathrm{mV}$, DC current $2 \mu \mathrm{A}$. Inset: voltage dependence of measured and calculated rectified current.

E. Frequency dependence of the rectified current in the $\mathrm{THz}$ regime. Maximum signal is attributed to a power of $2.5 \mathrm{~mW}$ from the frequency multiplier. Inset: Level of the rectified current (reference $0.1 \mu \mathrm{A}$ ) at $106.1 \mathrm{GHz}$. Measurement performed here with $100 \%$ amplitude modulation of $\mathrm{MW}$ at $100 \mathrm{kHz}$. RBW=1 $\mathrm{kHz}$, sweep time $275 \mathrm{~ms}, 4$ video averages. 

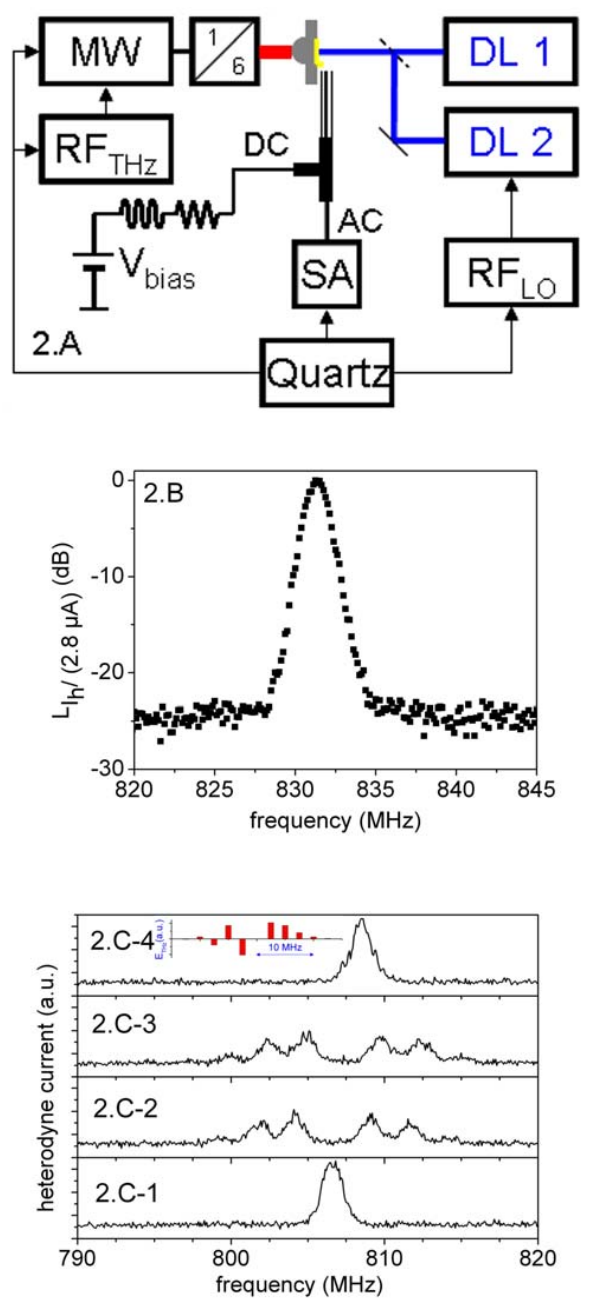

Figure 2: A. Experimental setup for heterodyne detection.

B. Level of the heterodyne beatnote (reference $2.8 \mu \mathrm{A}$ ) with $\mathrm{RBW}=1 \mathrm{MHz}$, sweep time $2.5 \mathrm{~ms}, 10$ video averages. Measurement at $100.1 \mathrm{GHz}$ with $\sim 0.9$ $\mathrm{mW}$. Bias voltage $40 \mathrm{mV}$, combined optical power $2 \times 20 \mathrm{~mW}$, DC current 2 $\mu \mathrm{A}$.

C. Multi-heterodyne beats between the $\mathrm{THz}$ source at $89.52 \mathrm{GHz}$ and the twolaser LO with 2 x $16 \mathrm{~mW}$ combined optical power.

-1 no modulation;

-2 only $\mathrm{THz}$ modulation at $2.5 \mathrm{MHz}$ with $R F_{T H z}$;

-3 only laser modulation at $2.5 \mathrm{MHz}$ with $R F_{L O}$;

$-4 \mathrm{THz}$ and laser modulation at the optimum phase and amplitude of $R F_{L O}$. RBW $1 \mathrm{MHz}$, sweep time $5 \mathrm{~ms}, 10$ video averages. 